ORIGINAL ARTICLE

Enhanced antitumor effect of combining TRAIL and MnSOD mediated by CEA-controlled oncolytic adenovirus

\title{
in lung cancer
}

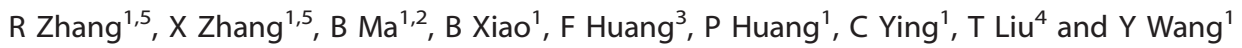

\begin{abstract}
Lung cancer, especially adenocarcinoma, is one of the leading causes of death in the world. Carcinoembryonic antigen (CEA), a superb non-small-cell lung cancer marker candidate, showed a beneficial effect in cancer therapy with oncolytic adenovirus in recent studies. Cancer-targeting dual gene-virotherapy delivers two therapeutic genes, linked by a connexon, in the replicationdeficient vector instead of one gene so that they can work in common. In this study, we constructed a tumor-specific oncolytic adenovirus, CD55-TRAIL-IETD-MnSOD. The virus has the fusion protein complementary DNAs for tumor necrosis factor-related apoptosis-inducing ligand (TRAIL) and for manganese superoxide dismutase (MnSOD) complementary DNA linked through a 4-amino acid caspase-8 cleavage site (IETD), and uses a CEA promoter to control virus E1A express. This is the first work to use a CEA promoter-regulated oncolytic adenovirus carrying two therapeutic genes for cancer research. Its targeting and anticancer capacity was evaluated by in vitro and in vivo experiments. The results indicated that CD55-TRAIL-IETD-MnSOD caused more cell apoptosis than CD55-TRAIL or CD55-MnSOD alone, or their combination in vitro, with low cytotoxicity of normal cells. In the A549 tumor xenograft model in nude mice, data showed that CD55-TRAIL-IETD-MnSOD could effectively suppress tumor growth than single gene groups, with no histological damage in liver, spleen or kidney tissues. Thus, the CEA-regulated dual-gene oncolytic virus CD55-TRAIL-IETD-MnSOD may be a novel potential therapy for lung cancer.
\end{abstract}

Cancer Gene Therapy (2016) 23, 168-177; doi:10.1038/cgt.2016.11; published online 15 April 2016

\section{INTRODUCTION}

In China, lung cancer incidence and mortality have increased during the past few decades. ${ }^{1}$ The morbidity of lung cancer has increased at the rate of $26.9 \%$ per year to become the leading cause of cancer-related death in our country. Therefore, the exploration of novel and effective therapy methods for lung cancer is imperative.

Cancer-targeting gene-virotherapy (CTGVT) is a novel strategy of combined gene therapy and virotherapy together that is constructed by inserting an antitumor gene into an oncolytic viral vector $(\mathrm{OV}){ }^{2,3}$ An oncolytic viral vector, such as the commonly used ZD55 in our group, instead of a replication-deficient vector, can target and replicate several hundred-fold in cancer cells, leading to several hundred-fold expression of the inserted gene in cancer cells. ${ }^{4}$ Therefore, the therapeutic effects of CTGVT (OVgene) have been significantly improved in recent publications. ${ }^{5}$ However, there remain some issues in CTGVT strategy such as the restricted targeting of $\mathrm{OV}$ and inefficient antitumor effects of a single antitumor gene. Consequently, the cancer-targeting dual gene-virotherapy (CTGVT-DG) strategy was exploited by us. ${ }^{6}$ Previous studies proved that the combination of ZD55-TRAIL and Ad-K5, ${ }^{7}$ or ZD55-TRAIL and ZD55-MnSOD, ${ }^{8}$ or ZD55-TRAIL and ZD-IL-24, ${ }^{8}$ as well as ZD55-TRAIL-IETD-Smac, ${ }^{9}$ co-expression of the therapeutic genes TRAIL and Smac by a linker had a much stronger antitumor effect in vitro and in vivo.
However, improving the tumor specificity of OV is another goal, and transcriptional targeting can be a crucial strategy to attain this goal. Carcinoembryonic antigen (CEA), a type of acidoglycoprotein with human embryo antigen specificity, is mainly expressed in fetal gastrointestinal epithelial tissue, pancreas and liver, but only at low levels in normal tissue. Its concentration is increased in colorectal cancer ${ }^{10-12}$ and gastric carcinoma. ${ }^{13}$ Furthermore, some studies have found that the serum CEA also increases in patients with lung cancer, ${ }^{14-16}$ and its level is also an important predictive factor for the postoperative survival rate in patients with nonsmall-cell lung cancer. ${ }^{17,18}$ These implied that CEA has the potential for targeting and modifying CTGVT in lung cancer.

Tumor necrosis factor-related apoptosis-inducing ligand (TRAIL) is a typical member of the tumor necrosis factor superfamily, and is currently being investigated as a potential therapeutic agent against many types of tumor cells via its death receptors and subsequent inducement of tumor cell apoptosis. ${ }^{19,20}$ Previous study reported that OV-mediated TRAIL could significantly suppress human hepatocellular carcinoma, ${ }^{21}$ lung adenocarcinoma ${ }^{22}$ and colorectal cancer. ${ }^{8}$ More than that, its combination with manganese superoxide dismutase (MnSOD) could completely eliminate a colorectal tumor xenograft ${ }^{23}$ via enhanced apoptosis. Some evidence also showed that MnSOD has effective antitumor ability in pancreatic, ${ }^{24}$ colorectal $^{25}$ and hepatocellular carcinoma. ${ }^{26}$ Based on the results using the separate OV-mediated delivery of

${ }^{1}$ Xinyuan Institute of Medicine and Biotechnology, School of Life Sciences, Zhejiang Sci-Tech University, Hangzhou, People's Republic of China; ${ }^{2}$ Department of Gastroenterology and Hepatology, Erasmus MC University Medical Center, Rotterdam, The Netherlands; ${ }^{3}$ School of Public health, Zhejiang University, Hangzhou, People's Republic of China and ${ }^{4}$ Institute of Biochemistry and Cell Biology, Shanghai Institutes for Biological Sciences, Chinese Academy of Sciences, Shanghai, People's Republic of China. Correspondence: Professor Y Wang, Xinyuan Institute of Medicine and Biotechnology, School of Life Sciences, Zhejiang Sci-Tech University, Hangzhou 310018, People's Republic of China. E-mail: wangyigang43@163.com

${ }^{5}$ These two authors contributed equally to this work.

Received 13 December 2015; revised 14 February 2016; accepted 16 February 2016; published online 15 April 2016 
Table 1. Sequences of primers

\begin{tabular}{|c|c|}
\hline Primers & Sequences \\
\hline \multirow[t]{2}{*}{ CEA } & Forward: 5'-CCGCTCGAGCTGGAGAGCATGGGGAGAC-3' \\
\hline & Reverse: 5'-TACGTAGTCTCTGCTGTCTGCTCTGT-3' \\
\hline \multirow[t]{2}{*}{ MnSOD } & Forward: 5'-AGTTGGCATTGAGACAGACAAGCACAGCCTCCCCGACC-3' \\
\hline & Reverse: 5'-CCGCTCGAGTTACTTTTTGCAAGCCAGT-3' \\
\hline \multirow[t]{2}{*}{ TRAIL-IETD } & Forward: 5'-GGAATTCATGGCTATGATGGAGGTCCA-3' \\
\hline & Reverse: 5'-GGTCGGGGAGGCTGTGCTTGTCTGTCTCAATGCCAACT-3' \\
\hline \multirow[t]{2}{*}{ Ad 5 virus } & Forward: 5'-CGCGGGAAAACTGAATAAGAG-3' \\
\hline & Reverse: 5'-ACCGCCAACATTACAGAGTCG-3' \\
\hline \multirow[t]{2}{*}{ CEA real-time $P C R$} & Forward: 5'-CTCCTGCTCACAGCCTCACTTC-3' \\
\hline & Reverse: 5'-TTCGGATCCACTCTTTTCACCTT-3' \\
\hline \multirow[t]{2}{*}{ GAPDH real-time PCR } & Forward: 5'-TTCGGATCCACTCTTTCACCTT-3' \\
\hline & Reverse: 5'-TGGAAGATGGTGATGGGAT-3' \\
\hline
\end{tabular}

Abbreviations: CEA, carcinoembryonic antigen; GAPDH, glyceraldehyde-3-phosphate dehydrogenase; MnSOD, manganese superoxide dismutase; TRAIL,

tumor necrosis factor-related apoptosis-inducing ligand.

TRAIL or MnSOD, it may be more effective to use one OV to deliver a bicistronic arrangement of both TRAIL and MnSOD. It has also been shown that the isoleucine-aspartate-threonine-glutamate (IETD) sequence encoding one of the caspase-8 cleavage sites forms a good link between two therapeutic genes, without affecting their function, and specifically the antitumor activity of TRAIL in the CTGV-DG strategy. ${ }^{9}$

In this study, we used the CEA promoter to regulate E1A expression of OV (ZD55) and first constructed a cancer-targeting dual gene-virus CD55-TRAIL-IETD-MnSOD carrying two therapeutic genes, TRAIL and MnSOD, linked by the tetrapeptide linker IETD. We further investigated the antitumor effect of the novel construct in lung adenocarcinoma for the first time. The experiments indicated that CD55-TRAIL-IETD-MnSOD could more effectively suppress tumor growth than either CD55-TRAIL or CD55-MnSOD or their combination, both in vivo and in vitro. The oncolytic virus enhanced tumor cell apoptosis and did not cause toxicity effects on normal cells.

\section{MATERIALS AND METHODS}

Cells and culture

Human lung bronchial epithelial cell line BEAS-2B and human embryonic kidney cell HEK293 (containing the E1A region of adenovirus) were obtained from ATCC (American Tissue Culture Collection, Rockville, MD, USA). Human colorectal cancer cell line SW480, normal human liver cell line QSG-7701, human hepatoma cell lines HepG2, Hep3B, Bel-7404 and SMMC-7721, human breast cancer cell line MCF-7, human gastric cancer cell line BGC-823, human cervical carcinoma cell line Hela and human lung carcinoma cell lines A549, NCl-H460, NCl-H1299 and NCl-H1975 were purchased from the Shanghai Cell Collection (Shanghai, China). The HEK293 and BEAS-2B were cultured in Dulbecco's modified Eagle's medium (GIBCO BRL, Grand Island, NY, USA) supplemented with $10 \%$ fetal bovine serum (GIBCO BRL). The cancer cell lines were cultured in Dulbecco's modified Eagle's medium supplemented with 5\% fetal bovine serum. All cell lines were incubated at $37^{\circ} \mathrm{C}$ in a humidified air atmosphere with $5 \% \mathrm{CO}_{2}$.

Plasmid and adenovirus construction

The adenovirus type 5 vector $p X C 2$, adenovirus packaging vector $\mathrm{pBHGE3}$, shuttle vector pShuttle, pCA13-TRAIL, pCA13-EGFP and pCA13-MnSOD were preserved in our laboratory. The oncolytic adenovirus plasmid pZD55 was constructed previously using site-directed mutation to delete the E1B 55-kDa gene. ${ }^{23}$ The novel shuttle vector pSD55 was obtained by inserting the expression cassette of pZD55 into pShuttle. The CEA promoter sequence was amplified by PCR from pGL3-CEAp. The PCR product was digested with Xholl and SnaBl, and then cloned into pXC2 to get the pXC2-CEA. Next, the CEA-E1A in pXC2-CEA was subcloned into pSD55 to obtain the pAdCEAp-D55 (briefly pCD55). The pCA13-TRAIL-IETD-
MnSOD was assembled and constructed from pCA13-TRAIL and PCA13MnSOD plasmids with the primer sequences of TRAIL-IETD and IETDMnSOD by overlap PCR. The PCR products were digested with Bg/ll and the TRAIL-IETD-MnSOD expression cassette was inserted into the corresponding site of pSD55 to obtain the pSD55-TRAIL/MnSOD. Similarly, pSD55TRAIL, pSD55-MnSOD and pSD55-EGFP were constructed. All the recombinant plasmids were confirmed by $P C R$, restriction enzyme digestion and DNA sequencing. All the primer sequences for PCR are shown in Table 1.

Generation, identification, purification and titration of adenovirus The oncolytic adenoviruses CD55-TRAIL, CD55-MnSOD, CD55-TRAIL/ MnSOD and CD55-EGFP were generated by homologous recombination in Escherichia coli strain, BJ-5183. Briefly, the shuttle plasmid pSD55-TRAIL, pSD55-MnSOD, pSD55-EGFP or pSD55-TRAIL/MnSOD and adenovirus packaging backbone plasmid pAdeasy-1 were co-transfected into BJ-5183, and the recombinant adenovirus genomes pSD55-TRAIL, pSD55-MnSOD, pSD55-EGFP or pSD55-TRAIL/MnSOD were obtained. After being digested with Pacl, each of them was transfected into HEK293 cells using Effectene transfection reagent (Qiagen, Germantown, MD, USA). After homologous recombination in HEK293 cells, the CEA-regulated E1B 55-kDa gene deletion oncolytic adenoviruses CD55-TRAIL, CD55-MnSOD, CD55EGFP and CD55-TRAIL/MnSOD carrying the TRAIL, MnSOD, EGFP and TRAIL/MnSOD genes, respectively, were generated. Each recombinant adenovirus was amplified by infecting HEK293 cells and purified by cesium chloride gradient ultracentrifugation. The virus titers were determined by the $50 \%$ tissue culture infectious dose $\left(\mathrm{TCID}_{50}\right)$ method in HEK293 cells and calculated as plaque-forming units (PFUs) per ml.

Assay of virus infection with reporter gene

Various cells were seeded in 24-well plates at a density of $5 \times 10^{4}$ cells per well and infected with CD55-EGFP at the indicated 0.1, 1 and 10 multiplicity of infection (MOI). After $48 \mathrm{~h}$, enhanced green fluorescent protein (EGFP) expression was detected using an Olympus fluorescence microscope with an Olympus digital camera apparatus DP70 (Tokyo, Japan).

\section{Quantitative real-time PCR}

Total RNA was isolated from prepared lung cancer cells or normal cells with TRIzol reagent (Invitrogen, Carlsbad, CA, USA) according to the manufacturer's instructions. For analysis of CEA expression, complementary DNA was synthesized by qPCR RT Kit (Toyobo, Osaka, Japan) as described by the manufacturer. Quantitative real-time PCR was performed using SYBR Green kit (Qiagen). The housekeeping gene, glyceraldehyde-3-phosphate dehydrogenase (GAPDH), was used for normalization. The primer sequences of CEA and GAPDH are shown in Table 1. Real-time PCR was run on the ABI Prism 7500 Sequence Detector (Applied Biosystems, Foster City, CA, USA). Each reaction was run in triplicate and repeated three times. We used the $\triangle \triangle C t$ method with relative quantification of gene expression to determine CEA mRNA expression levels. 


\section{Apoptotic cell staining and cell viability assay}

Cells were plated on 6-well plates and treated with various recombinant adenoviruses at $10 \mathrm{MOI}$. After $48 \mathrm{~h}$, cells were incubated with Hoechst 33342 (Beyotime, Nantong, China) for $10 \mathrm{~min}$, washed twice with phosphatebuffered saline (PBS) and observed under a fluorescence microscope. Cell viability was determined by methyl thiazolyl tetrazolium (MTT; SigmaAldrich, St Louis, MO, USA) assay according to the manufacturer's instructions (Biosharp, Hefei, Anhui, China) at the indicated MOI and time.

\section{Western blot analysis}

Cells were incubated with CD55-EGFP, CD55-MnSOD, CD55-TRAIL or CD55TRAIL-IETD-MnSOD at $10 \mathrm{MOI}$. After $48 \mathrm{~h}$, cell lysates were harvested and the protein concentration was determined by Pierce BCA protein assay kit (Thermo Fisher Scientific, Waltham, MA, USA). Then, an equal amount of total protein was subjected to SDS-polyacrylamide gel electrophoresis and transferred to polyvinylidene difluoride membranes after being boiled for $5 \mathrm{~min}$. The membranes were blocked with $5 \%$ nonfat dry milk and incubated with corresponding primary antibodies overnight. After incubation with peroxidase-conjugated secondary antibodies and enhanced chemiluminescence, each expressed protein was detected. Actin was used to control for equal gel loading. Caspase-8, caspase-3, caspase-9, poly-ADPribose polymerase, TRAIL, BAX and Bid antibodies were purchased from Cell Signaling Technology (Danvers, MA, USA). E1A, Actin and Smac antibodies were obtained from Santa Cruz Biotechnology (Santa Cruz, (A, USA).

\section{Flow cytometric analysis}

Cells were trypsinized and harvested after different treatment. Aliquots of cells were resuspended in $500 \mu \mathrm{l}$ of binding buffer and stained with Annexin V FITC/PI (BD Biosciences, San Jose, CA, USA) according to the manufacturer's instructions. A fluorescence-activated cell sorting (BD Biosciences) assay was performed immediately after staining.

\section{Animal experiment}

Animal experiments were performed according to the guide for regulations and standards of Experimental Animal of the US Department of Agriculture and the National Institutes of Health. Female BALB/c nude mice $\sim 4$ weeks old were purchased from the Shanghai Experimental Animal Center (Shanghai, China). A549 cells were injected subcutaneously into the right flank of nude mice to establish the tumor xenograft model.
When the tumors grew to $80-120 \mathrm{~mm}^{3}$ in size, mice were assigned to six groups $(n=8)$ at random. Different adenoviruses $\left(5 \times 10^{8}\right.$ PFUs) or PBS was injected once every other day for a total of four times in an intratumoral manner. From the end of the injection, tumor size was measured by the Vernier caliper every 5 days. The tumor volume $\left(\mathrm{mm}^{3}\right)$ was calculated as $\left(\right.$ length $\times$ width $\left.^{2}\right) / 2$. On day 7 after virus injection, tumors were harvested for histopathological and transmission electron microcopy, Immunohistochemistry $(\mathrm{IHC})$ and terminal deoxynucleotidyltransferase-mediated dUTP biotin nick end labeling (TUNEL) assays were performed.

\section{Histopathology, IHC and TUNEL assay}

Tumor tissue, liver, kidney and spleen were harvested and fixed in $4 \%$ paraformaldehyde, dehydrated with gradient ethanol and embedded in paraffin wax, and then cut in $5 \mu \mathrm{m}$ sections, dewaxed and rehydrated according to a standard protocol. For histopathology analysis, the sections were stained with hematoxylin and eosin. For IHC analysis, the tumor sections were incubated with rabbit monoclonal anti-TRAIL antibodies at 1:200 dilutions, washed with PBS and incubated with the avidin-biotinperoxidase complex reagent (Vector Laboratories, Burlingame, CA, USA) and detected with diaminobenzidine tetrahydrochloride solution containing $0.006 \%$ hydrogen peroxide. Hematoxylin was used as a counterstain. For the TUNEL assay, an in situ apoptosis detection kit (Sino-American Biotechnology Co., Luoyang, China) was used to stain in apoptotic cell tumor tissue sections according to the manufacturer's instructions. All sections were counterstained with hematoxylin.

\section{Transmission electron microscopy analysis}

Tumor tissue was cut in $1 \mathrm{~mm}^{3}$ pieces, fixed with $2.5 \%$ glutaraldehyde overnight and washed with PBS three times 5 min each time. Then, $1 \%$ osmium tetroxide was used to fix the tumor again. After being rinsed in $0.1 \mathrm{M}$ phosphate buffer, all of the samples were dehydrated in a graded series of ethanol and propylene oxide washes, embedded in pure acetone with embedding buffer in a ratio of 1:2 at room temperature for 3 to $4 \mathrm{~h}$ and cured at different gradients of temperature and time. The sections were doublestained with uranyl acetate and lead citrate, examined and photographed with a Jeol JEM-1230 transmission electron microscope (JEOL Ltd, Akishima, Japan).

\section{Statistical analysis}

All experimental data were presented as mean \pm s.d. Statistical analysis was performed using a one-way analysis of variance and compared at a given

Ad5 (WT) \begin{tabular}{|l|l|l|l|l|}
\hline \multicolumn{3}{c}{ Promoter (WT) } \\
\cline { 2 - 4 }
\end{tabular}

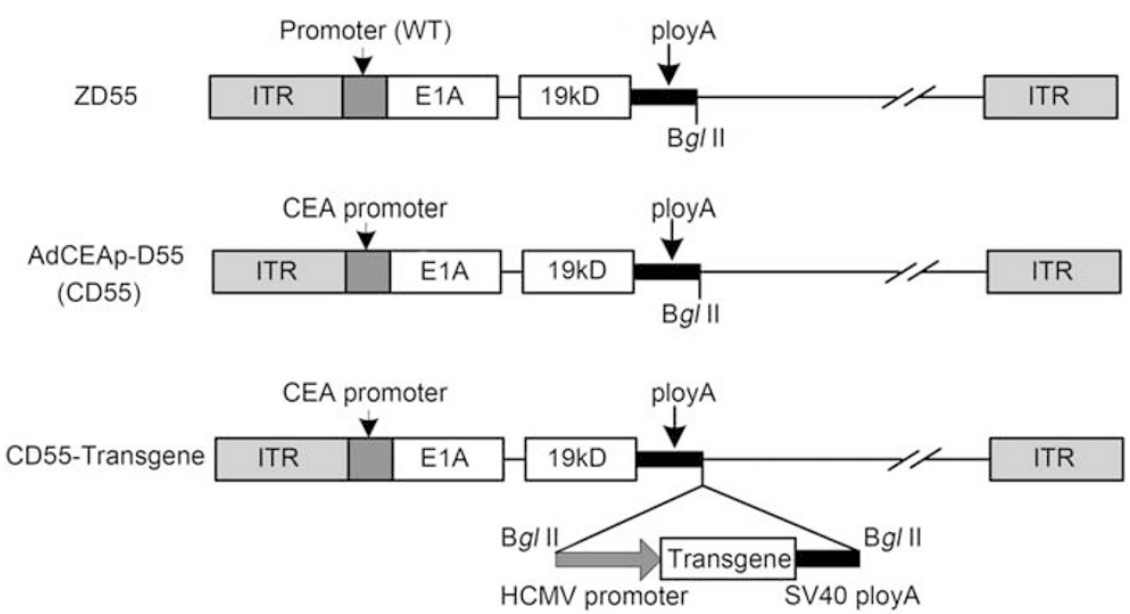

Figure 1. Schematic diagram of recombinant oncolytic adenovirus CD55 transgene. The transgenes included EGFP, TRAIL, MnSOD and TRAIL/ MnSOD. The wild-type adenovirus 5 was used as the backbone. EGFP, enhanced green fluorescent protein; ITR, inverted terminal repeat; MnSOD, manganese superoxide dismutase; TRAIL, tumor necrosis factor-related apoptosis-inducing ligand. 
condition by Student's $t$-test. Results were considered to be statistically significant when ${ }^{*} P<0.05$, to be very significant when ${ }^{* *} P<0.01$ and to be highly significant when ${ }^{* *} P<0.001$.

\section{RESULTS}

Construction and characterization of oncolytic adenovirus

Based on the study of CTGVT-DG, we constructed a novel oncolytic adenovirus CD55-TRAIL-IETD-MnSOD. The novelty displays three characteristics, including the CEA promoter regulating E1A gene, E1B 55-kDa gene deletion and delivery of the bicistronic genes TRAIL and MnSOD. The control single gene oncolytic viruses CD55-TRAIL, CD55-MnSOD and CD55-EGFP were also constructed for comparison (Figure 1).

To verify the CEA characteristic, we used real-time quantitative $P C R$ to detect the relative expression in a variety of cancer cell lines. The results showed that the expression of CEA mRNA was enhanced in SW480, Hep3B and HepG2 cell lines, especially in the A549 cell line (Figure 2a). Similarly, more EGFP expression mediated by the CEA-regulated oncolytic adenovirus CD55-EGFP was also observed in A549 cells than in Hep3B or SW480 cells in a dose-dependent manner, but minimally in the normal cell QSG-7701(Figure 2b). In addition, infected A549 cells with CD55EGFP, CD55-MnSOD, CD55-TRAIL, CD55-MnSOD plus CD55-TRAIL and CD55-TRAIL-IETD-MnSOD at the indicated MOI displayed a significant production of adenovirus protein E1A, and the transgenes TRAIL and MnSOD protein (Figure 2c).

a

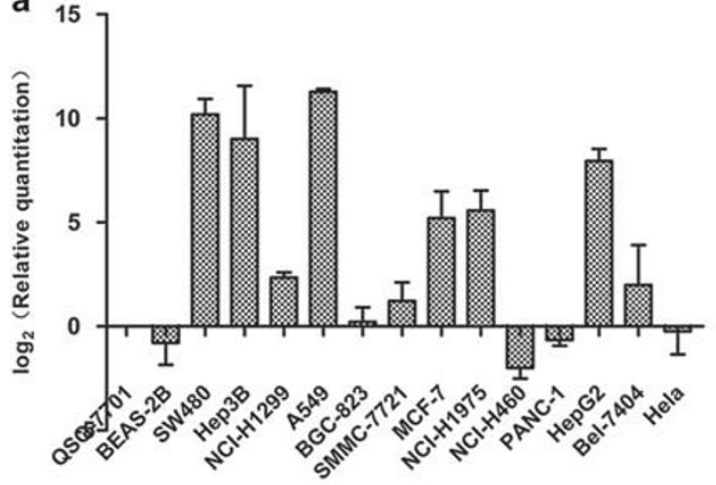

C

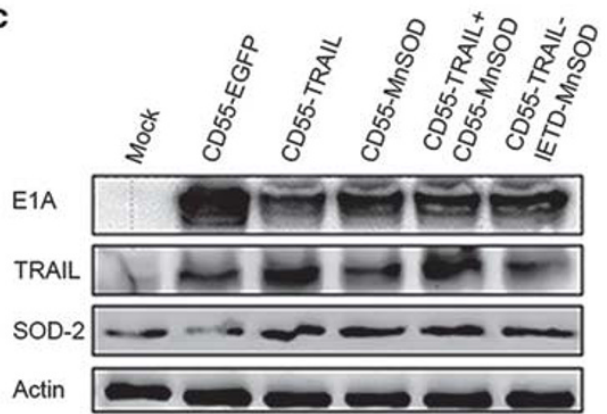

Tumor-selective cytopathic effect of CD55-TRAIL-IETD-MnSOD

To investigate the tumor-specific cytopathic effects, as well as to confirm the toxicity results, various human lung carcinoma cell lines and human lung normal cell line BEAS-2B were transduced with different OVs CD55-MnSOD, CD55-TRAIL and CD55-TRAILIETD-MnSOD at an MOI of 10, and combined treatment with CD55MnSOD and CD55-TRAIL each at an MOI of 5 . The results by MTT assay showed $\sim 40-70 \%$ cell death in cancer cell lines $96 \mathrm{~h}$ after infection with CD55-TRAIL-IETD-MnSOD, whereas BEAS-2B cells showed little cell death after infection with any of the viruses (Figure 3a). Among all lung cancer cells, CD55-TRAIL-IETD-MnSOD exhibited the most cytotoxic effect in A549 cells. Furthermore, cytopathic effect was examined in A549 cells by staining with crystal violet. CD55-TRAIL-IETD-MnSOD induced the most obvious cytopathic effect in tumor cells compared with the CD55-TRAIL or CD55- MnSOD or their combination (Figure 3b, left). Moreover, there was no distinct change in BEAS-2B cells after being transduced with different viruses (Figure $3 \mathrm{~b}$, right), suggesting that the CEA-regulated dual-gene-targeting oncolytic virus may not be toxic to normal cells.

Selective induction of cell apoptosis by CD55-TRAIL-IETD-MnSOD To further investigate whether the mechanism of the novel construct $\mathrm{OA}$ is mediated by apoptotic induction, we first performed the Hoechst assay to detect morphologic change of apoptosis on A549 cells. The result indicated that treatment with different viruses, especially the treatment with CD55-TRAIL-IETDMnSOD, resulted in apoptosis, characterized by chromatin condensation, nuclear fragmentation and the formation of apoptotic bodies (Figure 4a). Inspiringly, there was little apoptosis b

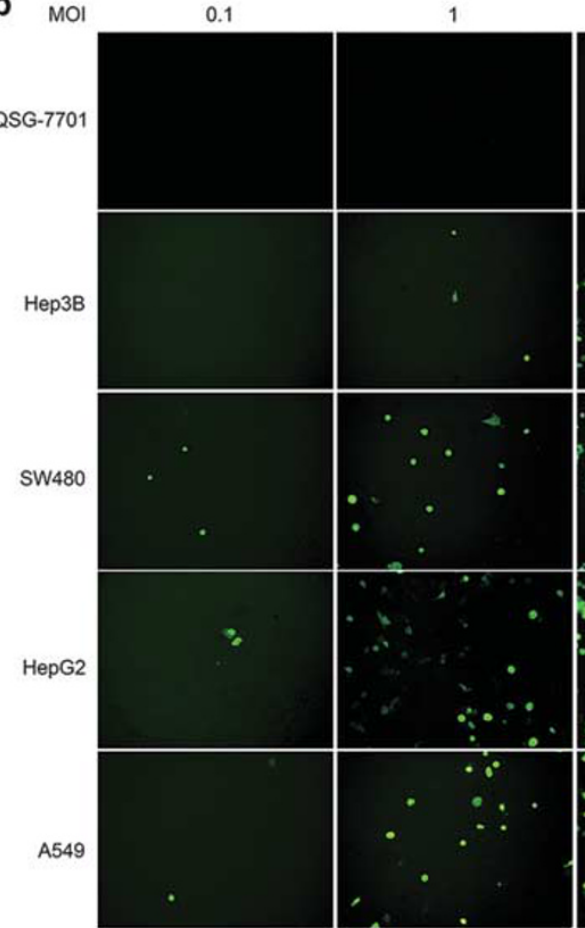

10

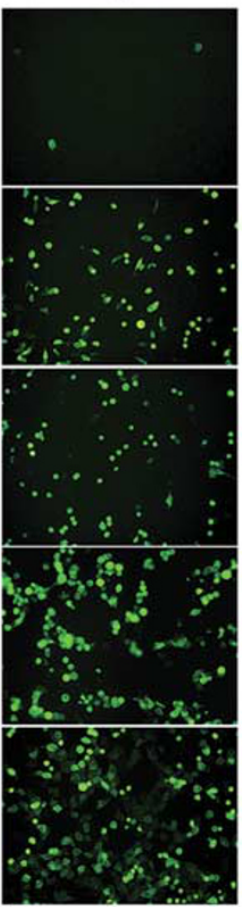

Figure 2. Characterization of oncolytic adenovirus CD55-TRAIL-IETD-MSnOD. (a) Real-time quantitative PCR (qPCR) to detect the relative background expression of carcinoembryonic antigen (CEA) in a variety of cancer cell lines. Glyceraldehyde-3-phosphate dehydrogenase (GAPDH) was used as an internal control. (b) Four high-expression CEA cancer cell lines, detected by qPCR, and the normal cell QSG-7701 were infected with different viruses at the indicated multiplicity of infection (MOl; $0.1,1$ and 10). After $72 \mathrm{~h}$, EGFP expression was observed by a fluorescence microscope. (c) Western blot was used to detect the E1A, TRAIL and MnSOD expression. A549 cells were infected with various viruses at $10 \mathrm{MOI}$, with untreated cells as controls. After $48 \mathrm{~h}$, cell lysates were prepared for analysis. Actin was used as protein loading control. EGFP, enhanced green fluorescent protein; MnSOD, manganese superoxide dismutase; TRAIL, tumor necrosis factor-related apoptosisinducing ligand. 

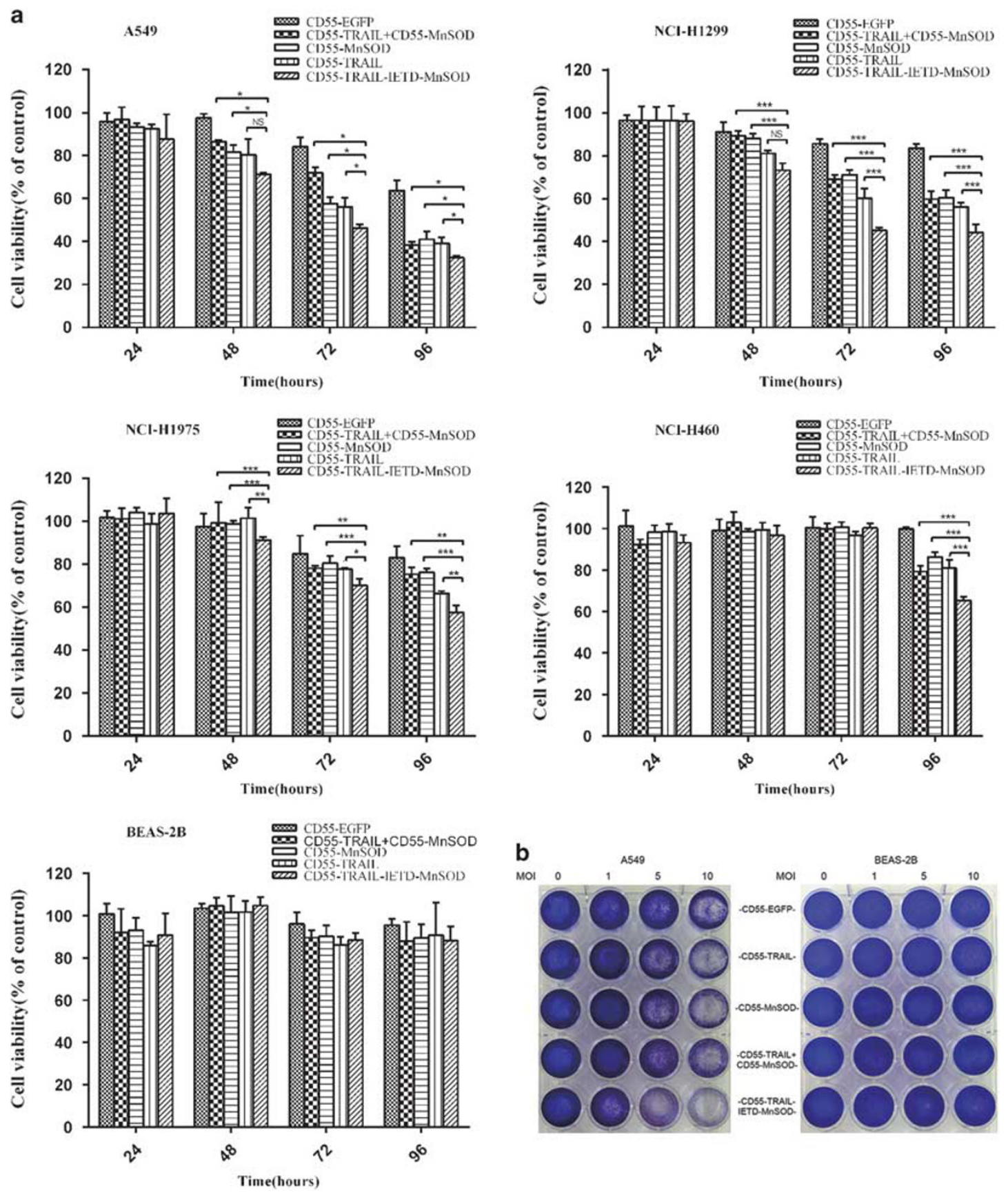

Figure 3. Tumor-selective cytopathic effect of CD55-TRAIL-IETD-MnSOD. (a) Four human lung carcinoma cell lines (A549, NCI-H460, NCI-H1299 and $\mathrm{NCl}-\mathrm{H} 1975$ ) and human lung bronchial epithelial cell line BEAS-2B were infected with CD55-EGFP, CD55-MnSOD, CD55-TRAIL and CD55TRAIL-IETD-MnSOD at the multiplicity of infection (MOI) of 10, or with CD55-TRAIL combined with CD55-MnSOD at an MOI of 5. After 24, 48, 72 or $96 \mathrm{~h}$ of infection, cell viability was measured by methyl thiazolyl tetrazolium (MTT) assay. There was no significant difference between each group in BEAS-2B cell lines. Data are presented as mean \pm s.d. $\left(n=3,{ }^{*} P<0.05,{ }^{* *} P<0.01,{ }^{* * *} P<0.0001\right)$. (b) The tumor cells A549 and the normal cells BEAS-2B were infected with different viruses at the indicated MOls; in combination therapy, the MOls of CD55-TRAIL and CD55-MnSOD were halved. After 4 days, cells were stained with crystal violet, and results are shown with the photograph. EGFP, enhanced green fluorescent protein; MnSOD, manganese superoxide dismutase; TRAIL, tumor necrosis factor-related apoptosis-inducing ligand.

in normal cells BEAS-2B. Similarly, apoptosis was also detected by flow cytometry assay in A549 cells (Figure 4b). Cells were infected with CD55-MnSOD, CD55-TRAIL, combined CD55-MnSOD with CD55-TRAIL (at a ratio of 1:1), and CD55-TRAIL-IETD-MnSOD at 10 $\mathrm{MOI}$ for $48 \mathrm{~h}$. Apoptosis in the treatment group with CD55-TRAIL plus CD55-MnSOD or with CD55-TRAIL-IETD-MnSOD was much greater than other groups. Consistent with the above, western blotting analysis showed a gradient decrease of procaspase-8, procaspase- 9 and procaspase- 3 in total protein when treated by CD55-EGFP, CD55-MnSOD, CD55-TRAIL, combination of CD55MnSOD and CD55-TRAIL as well as CD55-TRAIL-IETD-MnSOD, respectively (Figure $5 \mathrm{C}$ ). Furthermore, we observed an increased release of SOD-2 and Smac from mitochondria into the cytosol in cells treated with TRAIL/MnSOD compared with cells treated with 
a
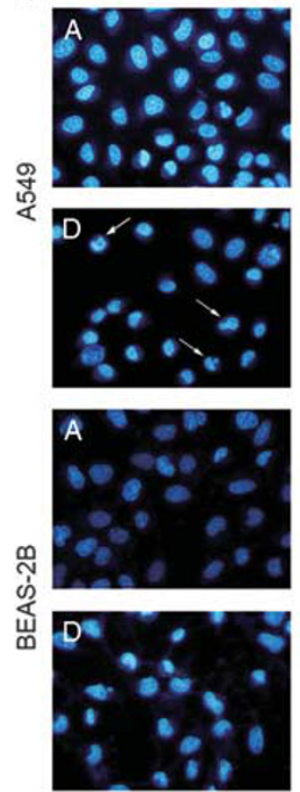
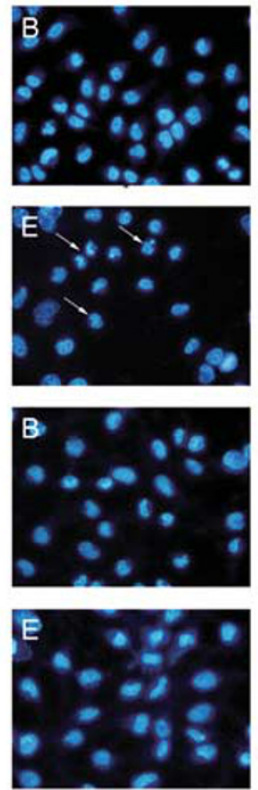
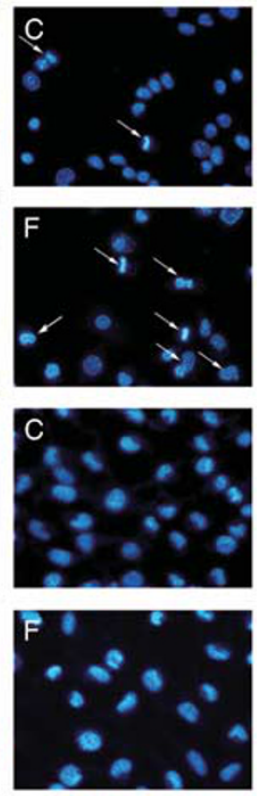

b
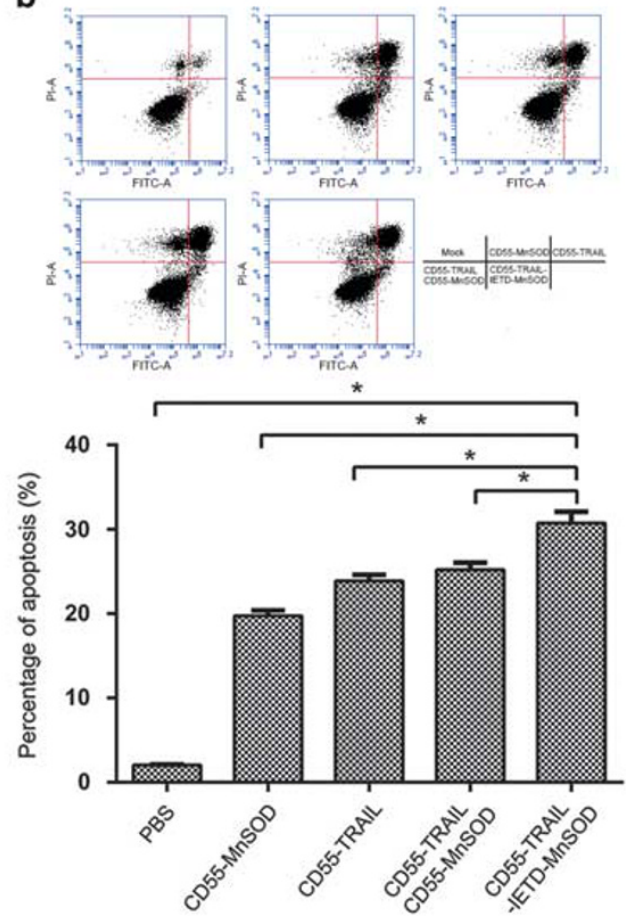

Figure 4. Selectively induced cell apoptosis and mechanism of action of CD55-TRAIL-IETD-MnSOD. (a) Cancer cells A549 (the upper two rows) and normal cells BEAS-2B (the lower two rows) were infected with (A) phosphate-buffered saline (PBS); (B) CD55-EGFP; (C) CD55-MnSOD; (D) CD55-TRAIL; (E) combination of CD55-TRAIL and CD55-MnSOD (at a ratio of 1:1); and (F) CD55-TRAIL-IETD-MnSOD at the multiplicity of infection (MOI) of 10 for $48 \mathrm{~h}$; apoptosis detection was by Hoechst 33342 staining. Chromatin condensation, nuclear shrinkage or nuclei fragmentation (arrows shown) were observed under the inverted fluorescence microscope (original magnification: $\times 200)$. (b) Flow cytometric analysis detected the percentage of apoptotic cells: treated A549 cells with different viruses at $10 \mathrm{MOl}$ for $48 \mathrm{~h}$; untreated group as control. Data are presented as mean \pm s.d. $\left(n=3,{ }^{*} P<0.05\right)$. EGFP, enhanced green fluorescent protein; MnSOD, manganese superoxide dismutase; TRAIL, tumor necrosis factor-related apoptosis-inducing ligand.
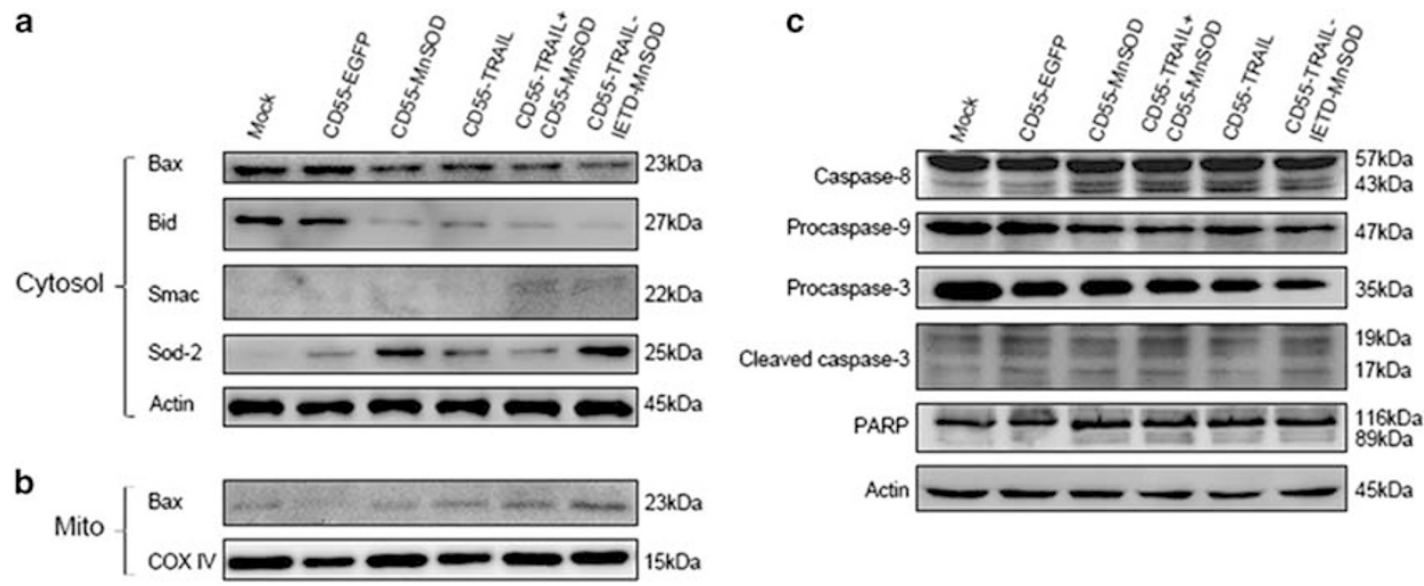

Figure 5. Western blot investigated the apoptosis-related protein expression. A549 cells were treated as previously described (see Figure 4 legend). The whole cytosol extracts (a), mitochondrial extracts (b) or cell extracts (c) were prepared and examined by western blot analysis. Actin or COX IV was used as protein loading control.

TRAIL or MnSOD alone or their combination (Figure 5a). The decrease of Bax or Bid in the cytosol (Figure 5a) and an obvious increase of Bax translocation from cytosol to mitochondria in the same group was also detected (Figure $5 \mathrm{~b}$ ). These findings suggest that the apoptosis resulting from TRAIL/MnSOD may depend on coordination of the caspase amplification loop and the mitochondrial pathway
Antitumoral efficacy of CD55-TRAIL-IETD-MnSOD in vivo

The data from the in vitro experiment indicated that CD55-TRAILIETD-MnSOD could cause lung carcinoma cell apoptosis and killing. The antitumor efficacy of CD55-TRAIL-IETD-MnSOD was also investigated in the A549 xenograft model in nude mice. As the tumor growth volumes show (Figure 6), the CD55-TRAIL-IETD-MnSOD treatment group had a significantly stronger antitumor effect 


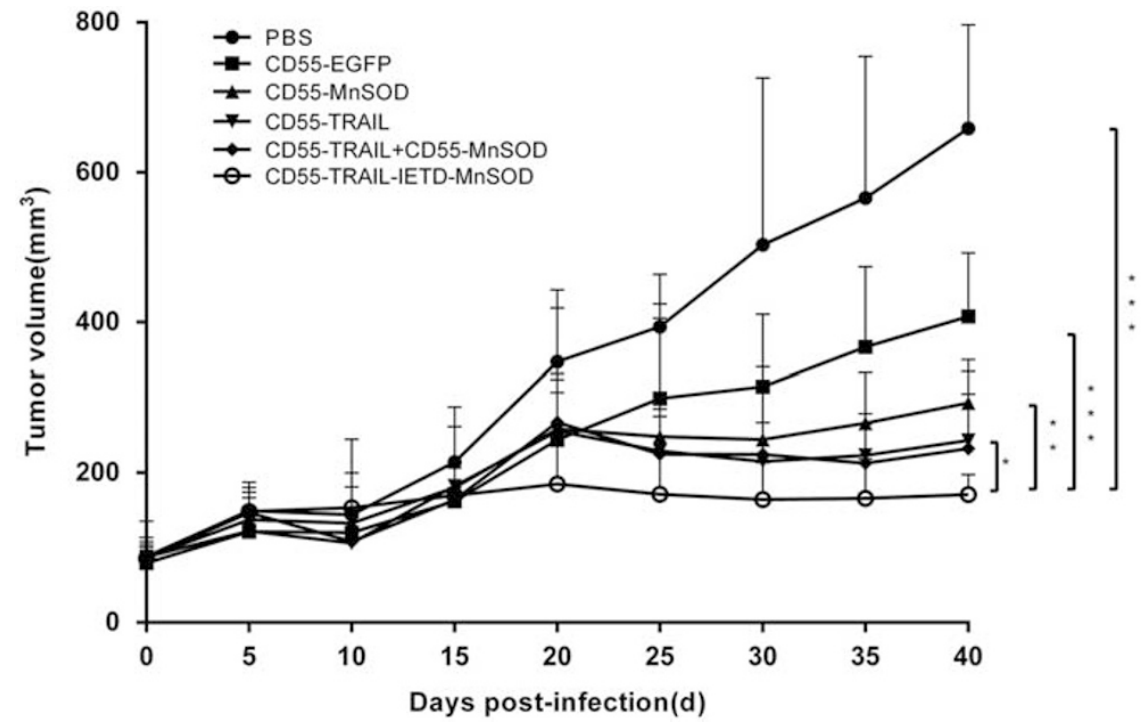

Figure 6. Antitumoral efficacy of CD55-TRAIL-IETD-MnSOD in vivo. A549 cells $\left(5 \times 10^{6}\right)$ were injected subcutaneously into female BALB/c nude mice to establish the tumor xenograft model. When the tumors grew to $80-120 \mathrm{~mm}^{3}$ in size, mice were assigned to six groups ( $n=8$ ) at random. Different adenoviruses $\left(5 \times 10^{8}\right.$ plaque-forming units (PFUs)) or phosphate-buffered saline (PBS) were injected once every other day for a total of four times. Tumor size was measured by the Vernier caliper every 5 days. The tumor volume $\left(\mathrm{mm}^{3}\right)=1 / 2\left(\right.$ length $^{3} \times$ width $\left.{ }^{2}\right)$. Data are presented as mean \pm s.d. $\left(n=8,{ }^{*} P<0.05,{ }^{*} P<0.01,{ }^{* * *} P<0.0001\right)$. MnSOD, manganese superoxide dismutase; TRAIL, tumor necrosis factor-related apoptosis-inducing ligand.

compared with CD55-TRAIL $(P<0.05)$ or CD55- MnSOD $(P<0.01)$, and the antitumor effect of CD55-TRAIL treatment group was similar to the combination group, CD55-TRAIL and CD55-MnSOD.

Furthermore, to detect the cell death and TRAIL expression in tumor tissues, we performed TUNEL, hematoxylin and eosin staining and IHC assays on day 7 after viral injection. TUNEL staining indicated that the CD55-TRAIL-IETD-MnSOD treatment group induced much more apoptosis than the others, and the combined group of CD55-TRAIL with CD55-MnSOD was observed to induce greater apoptosis than CD55-TRAIL or CD55-MnSOD alone (Figure 7a). Treatment with CD55-EGFP just showed a little apoptosis and no changes in the PBS group. Hematoxylin and eosin staining demonstrated that CD55-TRAIL-IETD-MnSOD caused more severe cytopathic effects than the other viruses (Figure 7a). Meanwhile, hematoxylin and eosin staining for some tissues, liver, kidney and spleen showed no obvious toxicity, implying an undetectable level of cell damage and apoptosis (Figure 7b). In addition, IHC staining demonstrated an enhanced expression of TRAIL in tumor tissues after treatment by both CD55-TRAIL-IETD-MnSOD and by CD55- MnSOD (Figure 7a). Thus, MnSOD could promote the expression of TRAIL protein in vivo.

Furthermore, transmission electron microcopy was also used to detect the representative morphological changes in tumor masses (Figure 8). All the treatment groups resulted in loss of tumor cell architecture except PBS, such as the appearance of nucleus deformation, nuclear collapse and chromatin agglutinated in lumps at the edge of nuclear envelope. However, the CD55-TRAILIETD-MnSOD group induced significant late apoptosis: vesicles from cytomembrane budding, increased blebbing of cytoplasm. All the results in vivo demonstrated that CD55-TRAIL-IETD-MnSOD could markedly suppress tumor growth and promote tumor cell apoptosis but induce no damage within normal tissues.

\section{DISCUSSION}

In this study, the dual-regulated oncolytic adenovirus vector CD55 that can replicate and induce tumor-specific cytotoxicity in CEApositive lung cancer cell lines with p53 dysfunction was designed based on CTGVT strategy. It has been suggested that the antitumor effect of TRAIL is limited by overexpression of inhibitors of apoptosis, tumor resistance to TRAIL, side effects and so on. ${ }^{27}$ Our previous study showed that the combination of MnSOD and TRAIL gene virotherapy could completely eliminate colorectal tumor xenografts, with their synergic effect mediated by their two separate oncolytic adenoviruses. ${ }^{23}$ Thus, in this report, we constructed a novel CEA-regulated dual-targeting oncolytic adenovirus harboring both the TRAIL and MnSOD genes (CD55TRAIL-IETD-MnSOD). In this construct, the four-peptide sequence encoding a caspase-8 cleavage site (IETD) was applied to mediate the double gene of TRAIL and MnSOD expression. We showed that this leads to equivalent amounts of TRAIL and MnSOD because caspase- 8 induced by TRAIL can cleave the TRAIL-IETD-MnSOD fusion protein. $8,9,28$ What is more, our group found that the cleaved TRAIL-IETD did not affect the function of TRAIL, ${ }^{9}$ suggesting the potent applied value of OV-mediated TRAIL-IETD gene, where the gene has a synergetic function with TRAIL.

Based on CTGVT-DG, our previous numerous studies found that the combined use of ZD55-TRAIL and ZD55 gene (K5, Smac, MnSOD, interleukin-24 and so on) have achieved complete suppression of xenograft tumors in colorectal cancer and hepatoma. ${ }^{8,9,23,29-33}$ Furthermore, our current data indicated that the treatment with CD55-TRAIL-IETD-MnSOD exerted much better antitumor effect than that of CD55-TRAIL alone, CD55-MnSOD alone or CD55-TRAIL plus CD55-MnSOD with halved dose, either as detected by MTT or crystal violet staining in vitro (Figure 3 ) or in mice in vivo (Figure 6). Our results further proved that CD55-TRAILIETD-MnSOD induced stronger lung cancer-specific cell apoptosis and caspase expression than CD55-TRAIL, CD55-MnSOD alone or their combination, as shown in Figure 5. Moreover, we found that injection of CD55-TRAIL-IETD-MnSOD did not cause obvious normal cell death in vitro, or hepatotoxicity, renal toxicity and spleen toxicity in vivo (Figure 7b), as detected by MTT methods and hematoxylin and eosin staining, respectively. The above data showed that our novel construct is an excellent candidate anticancer agent for lung cancer trials.

The use of the CEA promoter in our OVs is also novel. Currently, the core challenges in CTGVT strategy are the control of vector safety and choice of effective anticancer genes. Although the 
a

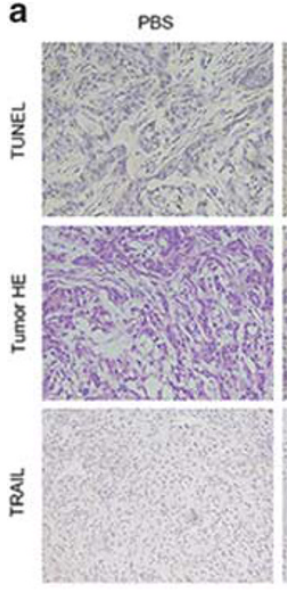

b
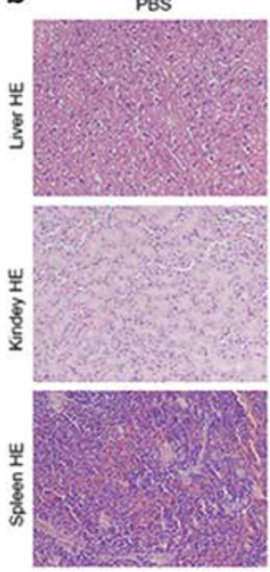

CO5S-EGFP
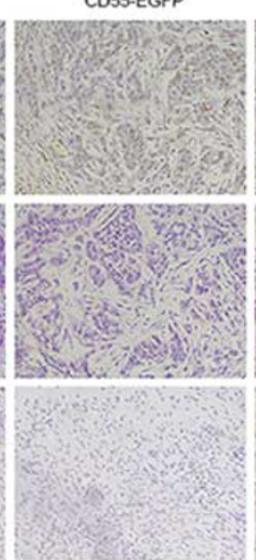

COSS-EGFP
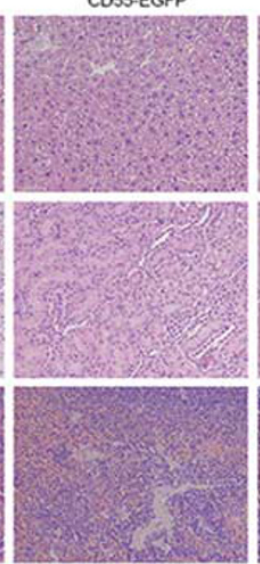

CDSS-MnSOO

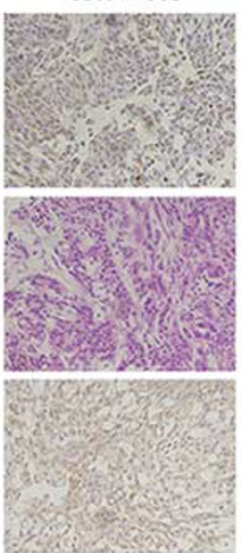

CDSs-Mnsoo

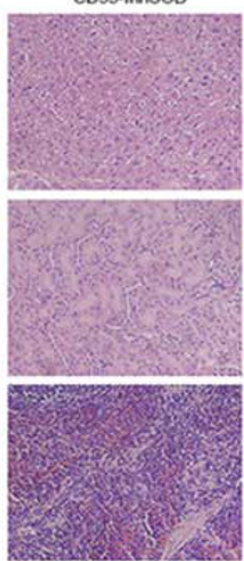

CDSS-TRAIL
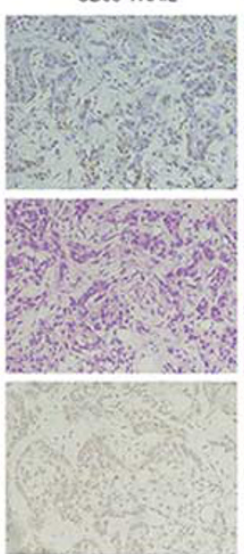

CDS5-TRAIL

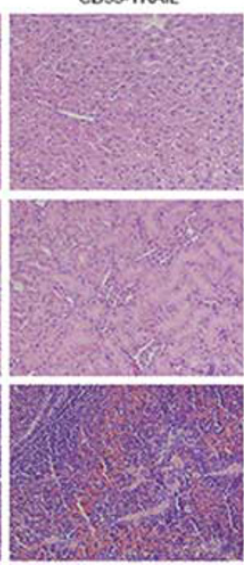

COS5-TRAIL+CDS5-MnSOD COS5-TRAIL-IETD-MnSOD

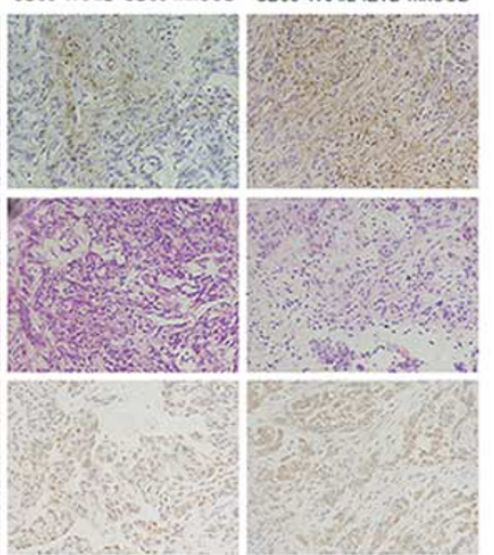

COS5-TRAIL +CDS5-MnSOD
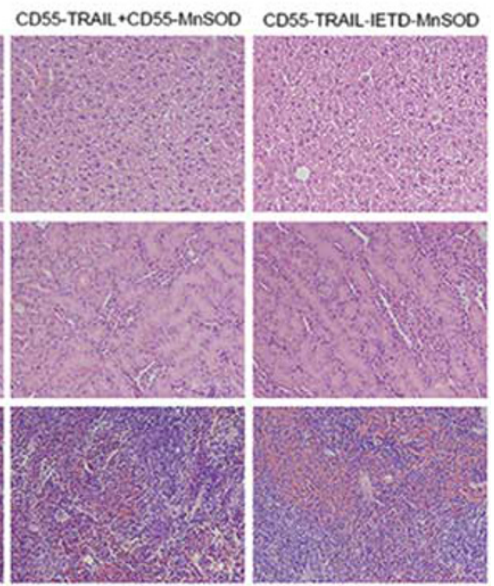

Figure 7. CD55-TRAIL-IETD-MnSOD induced cell death in vivo. Histological analysis of tumor sections or tissues from various treatments of A549 tumor xenografts. Samples were harvested at 7 days after infection. (a) The upper row shows TUNEL staining for detecting the apoptosis of tumor section; the middle row shows hematoxylin and eosin (HE) staining for cell necrotic areas in tumors; the lower row shows IHC staining for TRAIL expression of tumors tissues after transduction with different oncoviruses. (b) HE staining to detect toxicity of liver, kidney or spleen (original magnification: $\times 200$ ). IHC, immunohistochemistry; MnSOD, manganese superoxide dismutase; TRAIL, tumor necrosis factor-related apoptosis-inducing ligand; TUNEL, terminal deoxynucleotidyltransferase-mediated dUTP biotin nick end labeling.

CTGVT strategy has achieved excellent anticancer effects in the past decades by using an oncolytic adenovirus as the vector that could proliferate and replicate in tumor cells and cause the release of the progeny virus to infect, disrupt and kill more tumor cells, ${ }^{31-33}$ it also lacks the tumor specificity for some cancer types. For example, there is a lack of marker with high specificity for human lung cancer. Recent literature reported that CEA is positively expressed at high frequencies in lung cancer tissues, but not in normal lung tissues. ${ }^{14,34}$ Moreover, postoperative serum CEA level is a more significant prognostic factor than post/ preoperative level in non-small-cell cancer patients. ${ }^{35}$ The results suggested that CEA gene expression is closely related to lung cancer and provides a relatively broad spectrum of molecular targets for lung cancer gene therapy. Our data also showed that there is higher CEA mRNA expression in lung cancer cell lines than in normal cells (Figure 2). The CEA promoter was cloned to regulate the luciferase reporter gene in lung cancer cell lines, and promoter activity was detected by dual-luciferase reporter assay kit (data not shown). Therefore, we constructed a novel oncolytic adenovirus vector CD55 using the CEA promoter to regulate the adenovirus E1a gene. CD55-mediated EGFP fluorescent reporter gene expression in cancer cell lines was shown to gradually increase in a dose-dependent manner. However, in normal cell lines, low EGFP expression was observed, indicating that the novel
OV vector brought tumor-specific proliferating and replicating properties. Thus, specific OV proliferation and targeting expression of anticancer genes in lung cancer cells can be achieved using the CEA promoter to regulate the adenovirus vector carrying the TRAIL-IETD-MnSOD gene with low side effects in normal cells.

In the past few years, an increased interest has been focused on oncolytic virotherapy that made great progresses in the treatment of some malignant diseases. ${ }^{36}$ Besides the oncolytic adenovirus, the OVs have now expanded to include herpes simplex virus, vaccinia virus, coxsackie virus, respiratory and enteric orphan virus (reovirus), measles virus and so on. In particular, the first oncolytic virus agent (talimogene laherparepvec (T-VEC)) was authorized by the FDA (US Food and Drug Administration) and EFSA (European Food Safety Authority) because T-VEC (genetically modified herpes simplex virus expressing granulocyte-macrophage colonystimulating factor) improves durable response rate in patients with advanced melanoma phase III trial. ${ }^{37}$ In addition, many trials of OVs have focused on clinical end points, such as safety, response rate and overall survival, indicating a good prospect. ${ }^{36}$ Another significant enthusiasm for cancer therapy is precision medicine, cell and immunotherapy. Cancer immunotherapy, virotherapy and gene therapy were added as a fourth weapon after surgery, radiation and chemotherapy, with the intention of also decreasing risks of high mortality and/or causing adverse side 

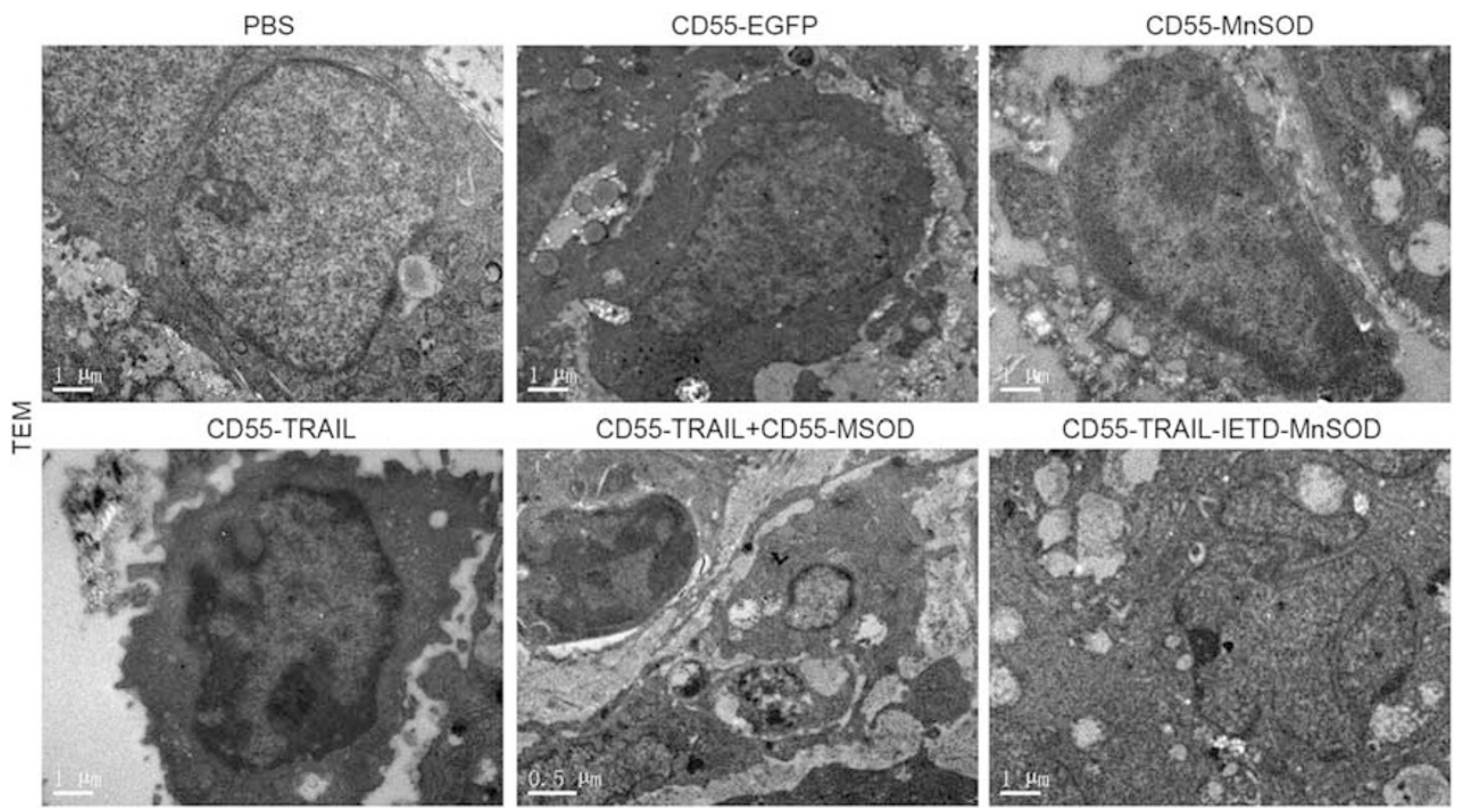

Figure 8. Morphological observation of A549 xenograft tumor tissue by transmission electron microscopy (TEM). Some obvious apoptotic phenomena, such as nucleus deformation, nuclear collapse and chromatin agglutinated in lumps at the edge of nuclear envelope, were observed in the treatment group but not in the control phosphate-buffered saline (PBS)-treated group. However, the CD55-TRAIL-IETDMnSOD group appeared to show significant late-stage apoptosis, as vesicles from cytomembrane budding and increased blebbing of cytoplasm. MnSOD, manganese superoxide dismutase; TRAIL, tumor necrosis factor-related apoptosis-inducing ligand.

effects. Successful clinical trials of CAR-T, ${ }^{38}$ anti-CTLA-4 and anti$\mathrm{PD}-1{ }^{39}$ and anti-PD-L $1^{40}$ therapy demonstrate such promising results in cancer patients that cancer immunotherapy was selected as the Breakthrough of the Year for 2013 and 2014. Of course, our OVs also may exert efficient cancer immunotherapy effect by carrying immune-associated genes, such as granulocytemacrophage colony-stimulating factor.

Although our novel CEA-regulated dual-targeting OVs have obtained strong antitumor effect in lung cancer cells and in animal models, there are still challenges requiring additional development. The cytotoxicity is still low in lung cancer cells after treatment by CD55-TRAIL-IETD-MnSOD, and it was also not able to completely eradicate tumor xenografts despite CD55-mediated TRAIL and MnSOD efficiently suppressing tumor growth in mice. These disadvantages might be mainly caused by host immune responses to viruses ${ }^{41}$ and the drug resistance to oncolytic viruses. ${ }^{42}$ Thus, it is urgent to solve these issues and achieve the significant therapeutic efficacy through combining conventional chemotherapy or radiotherapy as well as immunotherapy in future cancer therapy. ${ }^{43}$

In conclusion, our successfully engineered OV CD55, with the CEA promoter regulating the targeting oncolytic adenovirus vector, has strong elements regarding both the safety and effectiveness of our CTGVT strategy. This double-targeted CD55 can replicate specifically in CEA-positive lung cancer cells and can efficiently mediate dual anticancer gene TRAIL-IETD-MnSOD expression to maximize the oncolytic effect and suppression of tumor growth and minimize the side effects on normal cells and tissues. Thus, it has broad applications and can become an effective anticancer drug candidate for lung cancer therapy.

\section{CONFLICT OF INTEREST}

The authors declare no conflict of interest.

\section{ACKNOWLEDGEMENTS}

This work was supported by the National Nature Science Foundation of China (No. 81272687 and 81573000), Zhejiang Provincial Natural Science Foundation of China (No. LZ13H160004 and LY16H160056), Hi-Tech Research Development Program of China (863 Program, No. 2012AA020806), the Grant for 521 talent project of ZSTU, Zhejiang Province Health Department (No. 2014RCA022) and Technology Bureau of Hangzhou (20130733Q36).

\section{REFERENCES}

1 Chen W, Zhang S, Zou X. Estimation and projection of lung cancer incidence and mortality in China. Zhongguo Fei Ai Za Zhi 2010; 13: 488-493.

2 XY L. A new anticancer strategy-gene-virotherapy of cancer. Chin J Cancer Biother 2001; 8: 1 .

3 Zhang ZL, Zou WG, Luo CX, Li BH, Wang JH, Sun LY et al. An armed oncolytic adenovirus system, ZD55-gene, demonstrating potent antitumoral efficacy. Cell Res 2003; 13: 481-489.

4 Zhang Q, Nie M, Sham J, Su C, Xue H, Chua D et al. Effective gene-viral therapy for telomerase-positive cancers by selective replicative-competent adenovirus combining with endostatin gene. Cancer Res 2004; 64: 5390-5397.

5 Liu XY. The excellent anti-tumour strategy (CTGVT, OV-gene) and the excellent anti-tumor gene (IL-24). Int J Biome Sci 2012; 8: 87-93.

6 Liu XY. Targeting gene-virotherapy of cancer and its prosperity. Cell Res 2006; 16: 879-886.

7 Liu XY, Qiu SB, Zou WG, Pei ZF, Gu JF, Luo CX et al. Effective gene-virotherapy for complete eradication of tumor mediated by the combination of hTRAIL (TNFSF10) and plasminogen k5. Mol Ther 2005; 11: 531-541.

8 Zhao L, Dong A, Gu J, Liu Z, Zhang Y, Zhang W et al. The antitumor activity of TRAIL and IL-24 with replicating oncolytic adenovirus in colorectal cancer. Cancer Gene Ther 2006; 13: 1011-1022.

9 Wang SB, Tan Y, Lei W, Wang YG, Zhou XM, Jia XY et al. Complete eradication of xenograft hepatoma by oncolytic adenovirus ZD55 harboring TRAIL-IETD-Smac gene with broad antitumor effect. Hum Gene Ther 2012; 23: 992-1002.

10 Hang ZQ, Zheng MF, Huang JH. Detection and diagnostic value of serum carcinoembryonic antigen and cytokeratin 19 fragment in lung cancer patients. Zhonghua Zhong Liu Za Zhi 2011; 33: 847-849.

11 Verberne CJ, Nijboer CH, de Bock GH, Grossmann I, Wiggers T, Havenga K. Evaluation of the use of decision-support software in carcino-embryonic antigen (CEA)-based follow-up of patients with colorectal cancer. BMC Med Inform Decis Mak 2012; 12: 1-5. 
12 Wang YR, Yan JX, Wang LN. The diagnostic value of serum carcino-embryonic antigen, alpha fetoprotein and carbohydrate antigen 19-9 for colorectal cancer. $J$ Cancer Res Ther 2014; 10: 307-309.

13 Lai H, Jin Q, Lin Y, Mo X, Li B, He K. Combined use of lysyl oxidase, carcinoembryonic antigen, and carbohydrate antigens improves the sensitivity of biomarkers in predicting lymph node metastasis and peritoneal metastasis in gastric cancer. Tumour Biol 2014; 35: 10547-10554.

14 Grunnet M, Sorensen JB. Carcinoembryonic antigen (CEA) as tumor marker in lung cancer. Lung Cancer 2012; 76: 138-143.

15 Zhao W, Yu H, Han Z, Gao N, Xue J, Wang Y. Clinical significance of joint detection of serum CEA, SCCA, and bFGF in the diagnosis of lung cancer. Int $J$ Clin Exp Pathol 2015; 8: 9506-9511.

16 Yu DH, Li JH, Wang YC, Xu JG, Pan PT, Wang L. Serum anti-p53 antibody detection in carcinomas and the predictive values of serum p53 antibodies, carcinoembryonic antigen and carbohydrate antigen $12-5$ in the neoadjuvant chemotherapy treatment for III stage non-small cell lung cancer patients. Clin Chim Acta 2011; 412: 930-935.

17 Facchinetti F, Aldiqeri R, Aloe R, Bortesi B, Ardizzoni A, Tiseo M. CEA serum level as early predictive marker of outcome during EGFR-TKI therapy in advanced NSCLC patients. Tumour Biol 2015; 36: 5943-5951.

18 Takahashi Y, Horio H, Hato T, Harada M, Matsutani N, Kawamura M. Predictors of post-recurrence survival in patients with non-small-cell lung cancer initially completely resected. Intera Cardiovas Thorac Surg 2015; 21: 14-20.

19 Ashkenazi A, Pai RC, Fong S, Leung S, Lawrence DA, Marsters SA et al. Safety and antitumor activity of recombinant soluble Apo2 ligand. J Clin Invest 1999; 104: 155-162.

20 Pan G, O'Rourke K, Chinnaiyan AM, Gentz R, Ebner R, Ni J et al. The receptor for the cytotoxic ligand TRAIL. Science 1997; 276: 111-113.

$21 \mathrm{Ma} \mathrm{H}$, Liu Y, Liu S, Kung HF, Sun X, Zheng D et al. Recombinant adeno-associated virus-mediated TRAIL gene therapy suppresses liver metastatic tumors. Int $J$ Cancer 2005; 116: 314-321.

22 Shi J, Zheng D, Liu Y, Sham MH, Tam P, Farzaneh F et al. Overexpression of soluble TRAIL induces apoptosis in human lung adenocarcinoma and inhibits growth of tumor xenografts in nude mice. Cancer Res 2005; 65: 1687-1692.

23 Zhang Y, Gu J, Zhao L, He L, Qian W, Wang J et al. Complete elimination of colorectal tumor xenograft by combined manganese superoxide dismutase with tumor necrosis factor-related apoptosis-inducing ligand gene virotherapy. Cancer Res 2006; 66: 4291-4298.

24 Weydert C, Roling B, Liu J, Hinkhouse MM, Ritchie JM, Oberley LW et al. Suppression of the malignant phenotype in human pancreatic cancer cells by the overexpression of manganese superoxide dismutase. Mol Cancer Ther 2003; 2: 361-369.

25 Behrend L, Mohr A, Dick T, Zwacha RM. Manganese superoxide dismutase induces p53-dependent senescence in colorectal cancer cells. Mol Cell Biol 2005; 25: 7758-7769.

26 Huang F, Ma B, Wang Y, Xiao R, Kong Y, Zhou X et al. Targeting gene-virusmediated manganese superoxide dismutase effectively suppresses tumor growth in hepatocellular carcinoma in vitro and in vivo. Cancer Biother Radiopharm 2014; 29: 403-411.

27 Pan Q, Liu B, Liu J, Cai R, Liu X, Qian C. Synergistic antitumor activity of XIAP-shRNA and TRAIL expressed by oncolytic adenoviruses in experimental HCC. Acta Oncol 2008; 47: 135-144.
28 Srinivasula SM, Datta P, Fan XJ, Fernandes-Alnemri T, Huang Z, Alnemri ES. Molecular determinants of the caspase-promoting activity of Smac/DIABLO and its role in the death receptor pathway. J Biol Chem 2000; 275: 36152-36157.

29 Cai Y, Liu X, Huang W, Zhang K, Liu XY. Synergistic antitumor effect of TRAlL and IL-24 with complete eradication of hepatoma in the CTGVT-DG strategy. Acta Biochim Biophys Sin(Shanghai) 2012; 44: 535-543.

30 Pei Z, Chu L, Zou W, Zhang Z, Qiu S, Qi R et al. An oncolytic adenoviral vector of Smac increases antitumor activity of TRAIL against HCC in human cells and in mice. Hepatology 2004; 39: 1371-1381.

31 He G, Lei W, Wang S, Xiao R, Guo K, Xia Y et al. Overexpression of tumor suppressor TSLC1 by a survivin-regulated oncolytic adenovirus significantly inhibits hepatocellular carcinoma growth. J Cancer Res Clin Oncol 2012; 138: 657-670.

32 Pesonen S, Kanqasniemi L, Hemminki A. Oncolytic adenoviruses for the treatment of human cancer: focus on translational and clinical data. Mol Pharm 2011; 8: 12-28.

33 Wang $\mathrm{Y}$, Liu $\mathrm{T}$, Huang $\mathrm{P}$, Zhao $\mathrm{H}$, Zhang $\mathrm{R}$, Ma B et al. A novel Golgi protein (GOLPH2)-regulated oncolytic adenovirus exhibits potent antitumor efficacy in hepatocellular carcinoma. Oncotarget 2015; 6: 13564-13578.

34 Arrieta O, Villarreal-Garza C, Martinez-Barrera L, Morales M, Dorantes-Gallareta Y, Pena-Curiel $\mathrm{O}$ et al. Usefulness of serum carcinoembryonic antigen (CEA) in evaluating response to chemotherapy in patients with advanced non small-cell lung cancer: a prospective cohort study. BMC Cancer 2013; 13: 254.

35 Tomita M, Ayabe T, Chosa E, Nakamura K. Postoperative serum CEA level is a more significant prognostic factor than post/preoperative serum CEA ratio in non-small cell cancer patients. Asian Pac J Cancer Prev 2015; 16: 7809-7812.

36 Turnbull S, West EJ, Scott KJ, Appleton E, Melcher A, Ralph C. Evidence for oncolytic virotherapy: where have we got to and where are we going? Viruses 2015; 7: 6291-6312.

37 Andtbacka RH, Kaufman HL, Collichio F, Amatruda T, Senzer N, Chesney J et al. Talimogene laherparepvec improves durable response rate in patients with advanced melanoma. J Clin Oncol 2015; 33: 2780-2688.

38 Holohan DR, Lee JC, Bluestone JA. Shifting the evolving CAR T cell platform into higher gear. Cancer Cell 2015; 28: 401-402.

39 Das R, Verma R, Sznol M, Boddupalli CS, Gettinger SN, Kluger H et al. Combination therapy with anti-CTLA-4 and anti-PD-1 leads to distinct immunologic changes in vivo. J Immunol 2015; 194: 950-959.

40 Powles T, Eder JP, Fine GD, Braiteh FS, Loriot Y, Cruz C et al. MPDL3280A (anti-PDL1) treatment leads to clinical activity in metastatic bladder cancer. Nature 2014; 515: $558-562$.

41 Wang W, Ji W, Hu H, Ma J, Li X, Mei W et al. Survivin promoter-regulated oncolytic adenovirus with $\mathrm{Hsp70}$ gene exerts effective antitumor efficacy in gastric cancer immunotherapy. Oncotarget 2014; 5: 150-160.

42 Lei W, Liu HB, Wang SB, Zhou XM, Zheng SD, Guo KN et al. Tumor suppressor in lung cancer-1 (TSLC1) mediated by dual-regulated oncolytic adenovirus exerts specific antitumor actions in a mouse model. Acta Pharmacol Sin 2013; 34: 531-540.

43 Ma B, Wang Y, Zhou X, Huang P, Zhang R, Liu T et al. Synergistic suppression effect on tumor growth of hepatocellular carcinoma by combining oncolytic adenovirus carrying XAF1 with cisplatin. J Cancer Res Clin Oncol 2015; 141 419-429. 